Marquette University

e-Publications@Marquette

$2-2018$

\title{
Emotion Regulation Deficits in Persons with Body-Focused Repetitive Behavior Disorders
}

Jennifer R. Alexander

Marquette University

David C. Houghton

Texas A\&M University

Christopher C. Bauer

Marquette University

Heather C. Lench

Texas A\&M University

Douglas W. Woods

Marquette University, douglas.woods@marquette.edu

Follow this and additional works at: https://epublications.marquette.edu/psych_fac

Part of the Psychology Commons

\section{Recommended Citation}

Alexander, Jennifer R.; Houghton, David C.; Bauer, Christopher C.; Lench, Heather C.; and Woods, Douglas W., "Emotion Regulation Deficits in Persons with Body-Focused Repetitive Behavior Disorders" (2018).

Psychology Faculty Research and Publications. 335.

https://epublications.marquette.edu/psych_fac/335 
Marquette University

e-Publications@Marquette

\section{Psychology Faculty Research and Publications/College of Arts and Sciences}

This paper is NOT THE PUBLISHED VERSION; but the author's final, peer-reviewed manuscript. The published version may be accessed by following the link in th citation below.

Journal of Affective Disorders, Vol. 227 (February 2018): 463-470. DOI. This article is @ Elsevier and permission has been granted for this version to appear in e-Publications@Marquette. Elsevier does not grant permission for this article to be further copied/distributed or hosted elsewhere without the express permission from Elsevier.

\section{Emotion Regulation Deficits in Persons with Body-Focused Repetitive Behavior Disorders}

\section{Jennifer R. Alexander}

Department of Psychology, Marquette University, Milwaukee, WI

Department of Psychology, Texas A\&M University, College Station, TX

David C. Houghton

Department of Psychology, Texas A\&M University, College Station, TX

Christopher C. Bauer

Department of Psychology, Marquette University, Milwaukee, WI

Department of Psychology, Texas A\&M University, College Station, TX

Heather C. Lench

Department of Psychology, Texas A\&M University, College Station, TX

Douglas W. Woods

Department of Psychology, Marquette University, Milwaukee, WI

Department of Psychology, Texas A\&M University, College Station, TX 


\section{Abstract \\ Background}

Conceptualizations of emotion dysregulation (ED) and body-focused repetitive behavior disorders (BFRBDs) imply that ED may be a central component of BFRBDs as well as a factor that distinguishes BFRBDs from nonimpairing, subclinical body-focused repetitive behaviors (BFRBs). The current study empirically tested these observations.

\section{Methods}

One hundred thirty-eight undergraduates (of 1900 who completed a screening survey) completed self-report measures assessing four emotion regulation (ER) deficits hypothesized to underlie ED (alexithymia, maladaptive emotional reactivity, experiential avoidance, and response inhibition when distressed); 34 of these participants had BFRBDs, 64 had subclinical BFRBs, and 42 were unaffected by BFRBs.

\section{Results}

Results indicated that participants with BFRBDs reported higher levels of maladaptive emotional reactivity, experiential avoidance, and response inhibition when distressed than participants with subclinical BFRBs and participants unaffected by BFRBs. These results held even when controlling for comorbidity and total number of reported BFRBs. Participants did not differ on alexithymia.

\section{Limitations}

Limitations of the current study include the BFRB groups' different distributions of BFRB types (e.g., hair pulling versus skin picking), the sample's demographic uniformity, and the fact that negative affectivity was not controlled when exploring BFRB group differences on ER deficits. Future research should improve on these limitations.

\section{Conclusions}

The current results suggest that ED is a factor that differentiates BFRBDs from subclinical BFRBs. Such results may be useful for generating hypotheses regarding mechanisms responsible for BFRBs' development into BFRBDs. Furthermore, these results may provide insight into factors that explain the efficacy of more contemporary behavioral treatments for BFRBDs.

\section{Keywords}

Body-focused repetitive behavior disorders, Body-focused repetitive behaviors, Emotion dysregulation

\section{Introduction}

Body-focused repetitive behaviors (BFRBs), such as hair pulling, skin picking, and nail biting, are habitual behaviors directed toward one's own body (Snorrason et al., 2012, Stein et al., 2008, Teng et al., 2004, Teng et al., 2002). Many individuals engage in subclinical (i.e., non-pathological) BFRBs, which can be defined as BFRBs that cause little-to-no functional impairment (Duke et al., 2009, Hayes et al., 2009, Stanley et al., 1995). However, some individuals engage in chronic and impairing BFRBs that persist despite attempts to stop (American Psychiatric Association [APA], 2013). Such patterns are known collectively as body-focused repetitive behavior disorders (BFRBDs; APA, 2013).

Emerging research suggests that emotion dysregulation (ED) may be a central aspect of BFRBDs and may also differentiate non-impairing BFRBs from recurrent and impairing BFRBDs (Gratz and Roemer, 2004, Gross, 2013). ED involves the continued use of emotion regulation (ER) strategies that may be effective in the short term but ultimately result in heightened emotional and physiological distress or contribute to physical, personal, or psychosocial impairment (Gratz and Roemer, 2004, Gross, 2013). It has been proposed that ED characterizes several psychiatric disorders. For instance, generalized anxiety disorder (GAD) is characterized by excessive use 
of worry as an ER strategy (Mennin et al., 2005), major depressive disorder (MDD) is characterized by excessive use of rumination as an ER strategy (Nolen-Hoeksema et al., 2008), and borderline personality disorder is frequently characterized by use of self-harm (e.g., self-cutting) as an ER strategy (Gratz and Roemer, 2004). Similarly, it has been hypothesized that BFRBDs involve ED (Roberts et al., 2013), as such disorders are characterized by the excessive use of BFRBs (American Psychiatric Association, 2013), and evidence indicates that BFRBs function to regulate emotions (Diefenbach et al., 2008, Diefenbach et al., 2002, Mansueto et al., 1997, Snorrason et al., 2010). Unfortunately, few studies have empirically examined ED in BFRBDs, and it remains unclear whether ED differentiates persons with BFRBDs from those with subclinical BFRBs and those without BFRBs.

\section{Outlining a model for examining maladaptive ER in BFRBDs}

Although there are several ways to analyze ED in psychiatric disorders, one particularly useful method involves examining the presence and magnitude of ER deficits, as such deficits may provide information about the presence and nature of ED (Gratz and Roemer, 2004). Various models suggest deficits in certain ER traits contribute to the manifestation of ED underlying psychiatric disorders, including alexithymia, maladaptive emotional reactivity, experiential avoidance, and ineffectual response inhibition when distressed (Gratz and Roemer, 2004, Mennin et al., 2005). Alexithymia consists of several different components, but those most obviously relevant to ER include the inability to (a) identify and understand emotional experiences and (b) distinguish between different types of internal phenomena (Taylor, 2000). Emotional reactivity is the sensitivity one has to certain emotional experiences; maladaptive emotional reactivity refers to abnormally heightened (hyperreactivity) or dampened (hyporeactivity) sensitivity to specific emotions (Berenbaum et al., 2003, Nock et al., 2008). Experiential avoidance involves the unwillingness to experience aversive internal events, including emotions ( (Hayes et al., 1996). Finally, ineffectual response inhibition when distressed is characterized by an inability to (a) inhibit prepotent responses (Oosterlaan et al., 1998) when distressed and/or (b) abstain from immediate goals that are detrimental to distal goals (Friedman and Miyake, 2004) when distressed. It is important to note that, in the Gratz and Roemer (2004) and Mennin et al. (2005) models of ED, the ability to demonstrate response inhibition when distressed is emphasized, but the role of general response inhibition in maladaptive ER is not discussed.

\section{ER deficits in BFRBDs}

Researchers have begun to explore alexithymia, maladaptive emotional reactivity, experiential avoidance, and ineffectual response inhibition when distressed in the context of various BFRBDs.

\subsection{Alexithymia}

Some research suggests that people affected by BFRBDs demonstrate higher levels of alexithymia than people unaffected by BFRBs. Indeed, two studies have found that persons affected by clinical skin picking reported higher levels of alexithymia than persons unaffected by clinical skin picking (Çalikuşu et al., 2002, Snorrason et al., 2010). In contrast, findings from a third study suggest that alexithymia deficits may be associated with simply having a BFRB rather than having a disorder, per se. Roberts et al. (2016) found that, relative to persons in their control group (i.e., persons unaffected by BFRBs), persons in their BFRB group (persons affected by subclinical or clinical BFRBs) reported higher levels of one component of alexithymia (i.e., lack of emotional clarity) but not another component of alexithymia (i.e., emotional awareness). However, considering that Roberts et al. (2016) did not distinguish between subclinical and clinical groups, interpreting these findings is difficult.

Additional research supports the notion that people with BFRBDs demonstrate higher levels of alexithymia. Rufer et al. (2014) observed that $15 \%$ of their hair pulling sample demonstrated higher levels of alexithymia than people in the general population. Furthermore, Rufer et al. (2014), as well as Arabatzoudis et 
al. (2017), observed a positive relationship between alexithymia and hair pulling severity. In contrast, one study did not observe a relationship between alexithymia and skin picking severity (Snorrason et al., 2010), but this study may have been underpowered to detect such a relationship.

\subsection{Maladaptive emotional reactivity}

Some research suggests that people affected by BFRBDs demonstrate higher levels of maladaptive hyperreactivity than people unaffected by BFRBDs. For instance, Snorrason et al. (2010) found that people with clinical skin picking reported higher levels of emotional hyperreactivity than people who were either affected by subclinical skin picking or unaffected by skin picking. Similarly, Wetterneck et al. (2016) found that persons with clinical hair pulling reported higher levels of emotional hyperreactivity than unaffected controls. In

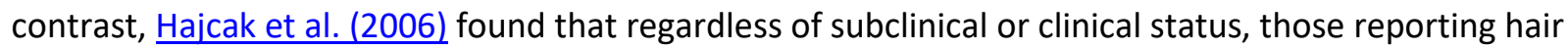
pulling/skin picking experienced higher levels of stress hyperreactivity relative to unaffected people. Such findings suggest that maladaptive emotional reactivity may be associated with simply having BFRBs, but such conclusions are tempered because the ratio of participants who experienced subclinical versus clinical BFRBs were not reported.

Although emotional hyperreactivity appears to be increased in persons with BFRBDs, there does not appear to be a clear relationship between BFRBD severity and level of emotional reactivity. Hajcak et al. (2006) found that stress hyperreactivity is positively related to hair pulling severity but not to skin picking severity. In addition, both Snorrason et al. (2010) and Wetterneck et al. (2016) failed to find significant relationships between skin picking severity and emotional reactivity. Thus, questions remain about whether emotional reactivity relates to BFRB severity.

\subsection{Experiential avoidance}

Although Roberts et al. (2016) were unable to conclude that people with clinical and/or subclinical BFRBs exhibit stronger repertoires of experiential avoidance than people without BFRBs, research more generally suggests that people with BFRBDs exhibit higher levels of experiential avoidance than people without BFRBDs. For example, Snorrason et al. (2010) found that people affected by clinical skin picking reported higher levels of experiential avoidance than people who were either affected by subclinical skin picking or were unaffected by skin picking. Similarly, Wetterneck et al. (2016) and Arabatzoudis et al. (2017)found that people affected by clinical hair pulling reported higher levels of experiential avoidance than unaffected controls. In addition, several studies suggest that experiential avoidance is positively related to hair pulling severity (Arabatzoudis et al., 2017, Begotka et al., 2004, Houghton et al., 2014, Norberg et al., 2007, Wetterneck et al., 2016) and skin picking severity (Flessner and Woods, 2006, Snorrason et al., 2010).

\subsection{Ineffectual response inhibition when distressed}

Evidence suggests that individuals with BFRBDs demonstrate response inhibition difficulties when distressed, relative to those without BFRBDs. Snorrason et al. (2010) found that persons affected by clinical skin picking reported having greater difficulty demonstrating response inhibition when distressed than did persons who were either affected by subclinical skin picking or were unaffected by skin picking. Similarly, Arabatzoudis et al. (2017) found that poorer response inhibition when distressed was related to greater hair pulling severity. Evidence also suggests that deficits in response inhibition when distressed may be associated with having both impairing and non-impairing BFRBs (Roberts et al., 2016).

\section{The current study}

With the exception of the Roberts et al. (2016) study, previous studies have only examined ER deficits in the context of one type of BFRBD (e.g., clinical hair pulling or clinical skin picking) at a time. Therefore, it is unclear 
whether observed ER deficits extend to all BFRBDs or if they are specific to certain types of BFRBDs.

Furthermore, previous studies typically have not compared the ER abilities of persons with BFRBDs to those with subclinical BFRBs, making it unclear whether ER deficits are specific to persons with BFRBDs or are specific to all persons with BFRBs.

The current study compared the ER deficits demonstrated by people with BFRBDs, people with subclinical BFRBs, and people unaffected by BFRBs. Based on previous research showing ER deficits in persons with BFRBDs and an extension of Gratz and Roemer's (2004) and Mennin et al.'s (2005) ED models to BFRBDs, it was hypothesized that people with BFRBDs would report higher levels of alexithymia, maladaptive hyperreactivity, experiential avoidance, and ineffectual response inhibition when distressed relative to those without BFRBs. Hypotheses were not made about whether there would be differences between the levels of ER deficits reported by people with subclinical BFRBs in comparison to people with BFRBDs and people unaffected with BFRBs, as these analyses were considered exploratory.

\section{Method}

\subsection{Sample}

Participants were undergraduate students who received partial course credit for their participation. Inclusion criteria specified that participants needed to be (a) enrolled in a psychology course in the university at which the study took place, (b) able to receive course credit for their participation in the study, and (c) at least 18 years old.

Prospective participants were required to complete an online screening survey. As part of this survey, participants indicated whether or not they had any of the following BFRBs: hair pulling, skin picking, nail biting, skin biting, teeth grinding, lip/mouth/cheek biting, and "other" BFRBs (e.g., nail picking). For each BFRB a participant endorsed, he or she was asked about the (a) the frequency of that BFRB, (b) the context in which that BFRB occurs, (c) the impairment and severity associated with that BFRB, and (d) the duration of that BFRB. Persons who reported both (a) engaging in at least one of the previously described BFRBs $\geq 5$ times a day over the course of at least one month and (b) experiencing impairment from the reported BFRB(s) were invited to complete the current study. Likewise, persons who reported that they did not engage in any BFRBs were invited to complete the current study. In total, 1900 undergraduate students completed this survey and agreed to be considered as potential participants for the current study, which consisted of an in-person interview. Of these, 138 were enrolled as participants in the current study. These participants' demographic information can be found in Table 1.

Table 1. Gender, psychiatric comorbidity, and age by BFRB group.

\begin{tabular}{|l|l|l|l|l|}
\hline Variables & $\begin{array}{l}\text { Control } \\
\text { group }\end{array}$ & $\begin{array}{l}\text { Subclinical BFRB } \\
\text { group }\end{array}$ & $\begin{array}{l}\text { BFRBD } \\
\text { group }\end{array}$ & Overall \\
\hline Gender & & & & \\
\hline Female & $28(67 \%)$ & $45(73 \%)$ & $24(71) \%$ & $97(70 \%)$ \\
\hline Male & $14(33 \%)$ & $17(27 \%)$ & $10(29 \%)$ & $41(30 \%)$ \\
\hline Race & & & & \\
\hline European American & $32(80 \%)$ & $50(82 \%)$ & $28(82 \%)$ & $110(82 \%)$ \\
\hline Black/African American & - & $6(10 \%)$ & $1(3 \%)$ & $7(5 \%)$ \\
\hline Asian/Asian American & $2(5 \%)$ & $2(3 \%)$ & $3(9 \%)$ & $7(5 \%)$ \\
\hline Native American or Native Alaskan & $1(3 \%)$ & - & $1(3 \%)$ & $2(2 \%)$ \\
\hline Other & $5(13 \%)$ & $3(5 \%)$ & $1(3 \%)$ & $9(7 \%)$ \\
\hline Ethnicity & & & & \\
\hline
\end{tabular}




\begin{tabular}{|l|l|l|l|l|}
\hline Hispanic & $10(24 \%)$ & $10(16 \%)$ & $3(9 \%)$ & $23(17 \%)$ \\
\hline Non-Hispanic & $32(76 \%)$ & $52(84 \%)$ & $31(91 \%)$ & $115(83 \%)$ \\
\hline $\begin{array}{l}\text { \% with current psychiatric disorder } \\
\text { other than BFRBD }\end{array}$ & $9(21 \%)$ & $26(42 \%)$ & $23(68 \%)$ & $58(42 \%)$ \\
\hline Age: $M(S D)$ & $19.22(4.07)$ & $19.15(2.58)$ & $18.85(1.12)$ & $19.10(2.87)$ \\
\hline
\end{tabular}

Each participant of the present study was categorized into one of the three following BFRB groups: BFRBD, subclinical BFRB, and control (unaffected by BFRBs). Categorization into each group was based on ratings on the Habit Disorders Interview (HDI). Created to measure Diagnostic and Statistical Manual 5th edition criteria for BFRBDs (APA, 2013) and given in a format mirroring the Trichotillomania Diagnostic Interview (TDI; Rothbaum and Ninan, 1994), the HDI was used to assess each participant's diagnostic status (i.e., clinical, subclinical, or unaffected) on seven types of BFRBs (i.e., hair pulling, skin picking, nail biting, skin biting, lip/mouth/cheek biting, teeth grinding, and "other"). BFRBs were rated "clinical" if they (a) had been done over the past week, (b) caused impairment (i.e., physical consequences and/or functional impairment or distress), and (c) had persisted despite attempts to stop or cut down on the behavior. BFRBs were rated "subclinical" if they (a) had been done over the past week and (b) had not caused impairment. Participants were categorized into the BFRBD group if they had at least 1 (of 7) BFRBs rated as "clinical." Participants were categorized into the subclinical BFRB group if they had at least 1 (of 7) BFRBs rated "subclinical" and did not have any BFRBs rated "clinical." Finally, participants were categorized into the control group if they had neither "clinical" nor "subclinical" BFRBs. Based on these classification criteria, 42 participants were categorized into the control group, 62 were categorized into the subclinical BFRB group, and 34 were categorized into the BFRBD group. Demographic information pertaining to each of these BFRB groups is presented in Table 1. In addition, information about the types of BFRBs endorsed by the subclinical BFRB and BFRBD groups are presented in Table 2.

Table 2. Number of persons in the subclinical BFRB and BFRBD groups reporting each type of BFRB.

\begin{tabular}{|l|l|l|l|l|}
\hline & $\begin{array}{l}\text { Subclinical BFRB } \\
\text { group }\end{array}$ & & BFRBD group & \\
\hline Types of BFRB & Subclinical-level & Clinical-level & Subclinical-level & Clinical-level \\
\hline Hair pulling & $n=10$ & - & $n=3$ & $n=1$ \\
\hline Skin picking & $n=28$ & - & $n=9$ & $n=13$ \\
\hline Nail biting & $n=29$ & - & $n=6$ & $n=9$ \\
\hline Cheek biting & $n=40$ & - & $n=14$ & $n=9$ \\
\hline Teeth grinding & $n=11$ & - & $n=7$ & $n=7$ \\
\hline Skin Biting & $n=8$ & - & $n=6$ & $n=3$ \\
\hline Other & $n=5$ & - & $n=5$ & $n=3$ \\
\hline
\end{tabular}

Note. Persons in the subclinical BFRB and BFRBD groups could report having more than one BFRB.

\subsection{Measures}

\subsubsection{Alexithymia}

Alexithymia was measured using two subscales on the Difficulties in Emotion Regulation Scale (DERS; Gratz and Roemer, 2004): the Awareness subscale (DERS-Awareness) and the Clarity subscale (DERS-Clarity). The DERSAwareness subscale consists of six items. Scores on this subscale range from 6 to 30; higher scores indicate a greater propensity to be inattentive to one's own emotional experiences. The DERS-Clarity subscale consists of five items. Scores on this subscale range from 5 to 25; higher scores indicate greater difficulty identifying and understanding one's own emotional experiences. Gratz and Roemer (2004)concluded that both the DERSAwareness and DERS-Clarity subscales demonstrate acceptable internal consistency, reliability, and validity in 
nonclinical samples. Further, both of these subscales have been utilized in BFRBD samples (e.g., Arabatzoudis et al., 2017; Roberts et al., 2016; Snorrason et al., 2010).

\subsubsection{Maladaptive emotional reactivity}

Maladaptive emotional reactivity was measured with the Personality AssessmentInventory Borderline Features- Affective Instability subscale (PAI-BOR-A; Morey, 2007, Morey, 1991), which is a six-item self-report subscale. $T$-scores of 50 on the PAI-BOR-A are representative of the average level of emotional reactivity reported by individuals included in a large community sample. $T$-scores higher than 50 represent increasingly pathological emotional hyperreactivity. The PAI-BOR-A has demonstrated acceptable reliability and validity in a variety of samples (Morey, 2007, Morey, 1991, Stein et al., 2007) including BFRBD samples (Wetterneck et al., 2016).

\subsubsection{Experiential avoidance}

Experiential avoidance was measured with the 7-item version of the Acceptance and Action QuestionnaireSecond Version (AAQ-II; Bond et al., 2011). Scores on the AAQ-II range from 7 to 49; higher scores indicate greater experiential avoidance. Bond et al. (2011) concluded that the AAQ-II demonstrates acceptable validity in a community sample. Moreover, the AAQ-II has been utilized in BFRBD samples (e.g., Houghton et al., 2014; Wetterneck et al., 2016).

\subsubsection{Response inhibition abilities when distressed}

Response inhibition abilities when distressed was measured with the five-item Goals subscale on the DERS (DERS-Goals) and the six-item Impulse subscale on the DERS (DERS-Impulse; Gratz and Roemer, 2004). Scores on the DERS-Goals subscale range from 5 to 25; higher scores indicate greater difficulty engaging in goaloriented thoughts and behavior when distressed. Scores on the DERS-Impulse subscale range from 6 to 30; higher scores denote greater difficulty maintaining control of behaviors when distressed. Gratz and Roemer (2004)concluded that the DERS-Goals and DERS-Impulse subscales demonstrate acceptable internal consistency, reliability, and validity in nonclinical samples. Further, these subscales have been utilized in BFRBD samples (e.g., Arabatzoudis et al., 2017; Roberts et al., 2016; Snorrason et al., 2010).

\subsubsection{General psychopathology}

The Mini-International Neuropsychiatric Interview (MINI 6.0; Sheehan et al., 1998) is a structured diagnostic interview of psychopathology. In the present study, the MINI was used to assess for the presence of psychiatric disorders other than BFRBDs. The MINI has demonstrated acceptable reliability and validity in various samples (Sheehan et al., 1998).

\subsection{Procedures}

Following consent, participants were evaluated with several instruments, including the HDI and the MINI. These evaluations were conducted by either a clinical psychology doctoral student or a master's-level research coordinator and were supervised by a licensed clinical psychologist. Following these evaluations, participants completed several questionnaires, including the DERS, AAQ-II, and PAI.

\section{Results}

\subsection{Preliminary analysis}

Preliminary analyses were conducted to examine whether age or gender should be entered as covariates in subsequent analyses. Results of a one-way ANOVA indicated the BFRB groups did not significantly differ on age, $F(2,131)=.17, p=.85$, and Pearson Product-Moment Correlations indicated that age was not significantly correlated with any of the variables of interest (see Table 3). Moreover, results of a chi-square analysis indicated that the BFRB groups did not differ on gender distributions, $\chi^{2}(2, N=138)=.42, p=.81$, and results of several $t$ - 
tests indicated that male and female participants did not demonstrate significant differences on any of the variables of interest (see Table 4 for males and females' means and standard deviations on variables of interest). Accordingly, neither age nor gender were included as covariates in subsequent analyses.

Table 3. Overall means, standard deviations, and correlations for variables of interest and age.

\begin{tabular}{|l|l|l|l|l|l|l|l|}
\hline Variables & $\mathbf{1}$ & $\mathbf{2}$ & $\mathbf{3}$ & $\mathbf{4}$ & $\mathbf{5}$ & $\mathbf{6}$ & $\mathbf{7}$ \\
\hline 1. DERS-Awareness & - & & & & & & \\
\hline 2. DERS-Clarity & $.46^{* *}$ & - & & & & & \\
\hline 3. PAI-BOR-A & .11 & $.48^{* *}$ & - & & & & \\
\hline 4. AAQ-II & $.20^{*}$ & $.65^{* *}$ & $.62^{* *}$ & - & & & \\
\hline 5. DERS-Goals & -.02 & $.39^{* *}$ & $.49^{* *}$ & $.60^{* *}$ & - & & \\
\hline 6. DERS-Impulse & $.20^{*}$ & $.55^{* *}$ & $.72^{* *}$ & $.69^{* *}$ & $.63^{* *}$ & - & \\
\hline 7. Age & .05 & .11 & .11 & .06 & .06 & .05 & - \\
\hline Mean & 15.44 & 11.47 & 51.10 & 19.97 & 14.25 & 11.17 & 19.10 \\
\hline Standard Deviation & 4.69 & 4.32 & 11.02 & 9.00 & 5.07 & 5.01 & 2.87 \\
\hline
\end{tabular}

Note. DERS-Awareness = Difficulties in Emotion Regulation Scale: Awareness Subscale; DERS-Clarity = Difficulties in Emotion Regulation Scale: Clarity Subscale; PAI-BOR-A = Personality Assessment Inventory Borderline Features-Affective Instability Subscale; $A A Q-I I=$ Acceptance and Action Questionnaire- Second Version; DERS-Goals = Difficulties in Emotion Regulation Scale: Goals Subscale; DERS-Impulse = Difficulties in Emotion Regulation Scale: Impulse Subscale.

${ }^{*} p<.05 .{ }^{* *} p<.01$.

Table 4. Gender differences on variables of interest.

\begin{tabular}{|l|l|l|l|}
\hline Variables & Females $\boldsymbol{M}(\boldsymbol{S D})$ & Males $\boldsymbol{M}(\boldsymbol{S D})$ & Effect size $(\boldsymbol{d})$ \\
\hline DERS-Awareness & $15.27(4.73)$ & $15.85(4.61)$ & -.12 \\
\hline DERS-Clarity & $11.71(4.37)$ & $10.90(4.19)$ & .19 \\
\hline PAI-BOR-A & $51.32(10.90)$ & $50.59(11.42)$ & .07 \\
\hline AAQ-II & $19.93(8.96)$ & $20.08(9.23)$ & -.02 \\
\hline DERS-Goals & $14.21(5.10)$ & $14.37(5.06)$ & -.03 \\
\hline DERS-Impulse & $11.10(5.19)$ & $11.32(4.62)$ & -.05 \\
\hline
\end{tabular}

Note. DERS-Awareness = Difficulties in Emotion Regulation Scale: Awareness Subscale; DERS-Clarity = Difficulties in Emotion Regulation Scale: Clarity Subscale; AAQ-II = Acceptance and Action Questionnaire- Second Version; PAI-BOR-A = Personality Assessment Inventory Borderline Features-Affective Instability Subscale; DERS-Goals = Difficulties in Emotion Regulation Scale: Goals Subscale; DERS-Impulse = Difficulties in Emotion Regulation Scale: Impulse Subscale.

Additional preliminary analyses were conducted to examine whether current comorbidity status or the number of BFRBs reported by participants should be entered as covariates in subsequent analyses. Results of a chisquare analysis indicated that the BFRB groups had significantly different comorbidity distributions, $\chi^{2}(2, N=$ $138)=16.47, p<.001$. In addition, results of several $t$-tests indicated that people with and without comorbid diagnoses demonstrated significantly different levels of emotional clarity $(p=.04)$, maladaptive hyperreactivity $(p=.001)$, experiential avoidance $(p<.001)$, ability to maintain goal-directed behavior when distressed $(p=$ $.002)$, and ability to maintain control of behaviors when distressed $(p<.001)$. See Table 5 for means and standard deviations on the variables of interest differentiated by comorbidity status.

Table 5. Comorbidity differences on variables of interest.

\begin{tabular}{|l|l|l|l|}
\hline Variables & Comorbid diagnosis $\boldsymbol{M ( S D )}$ & No comorbid diagnosis $\boldsymbol{M}(\boldsymbol{S D})$ & Effect size $(\boldsymbol{d})$ \\
\hline DERS-Awareness & $15.85(4.74)$ & $15.15(4.66)$ & .15 \\
\hline DERS-Clarity & $12.38(4.33)$ & $10.81(4.21)$ & .37 \\
\hline
\end{tabular}




\begin{tabular}{|l|l|l|l|}
\hline PAI-BOR-A & $55.72(11.46)$ & $47.81(9.47)$ & .75 \\
\hline AAQ-II & $23.47(9.04)$ & $17.41(8.12)$ & .71 \\
\hline DERS-Goals & $15.79(4.78)$ & $13.14(5.01)$ & .54 \\
\hline DERS-Impulse & $13.31(5.74)$ & $9.61(3.74)$ & .76 \\
\hline
\end{tabular}

Note. DERS-Awareness = Difficulties in Emotion Regulation Scale: Awareness Subscale; DERS-Clarity = Difficulties in Emotion Regulation Scale: Clarity Subscale; AAQ-II = Acceptance and Action Questionnaire- Second Version; PAI-BOR-A = Personality Assessment Inventory Borderline Features-Affective Instability Subscale; DERS-Goals = Difficulties in Emotion Regulation Scale: Goals Subscale.

Results of a $t$-test indicated that, on average, the BFRBD group $(M=2.82, S D=1.38)$ reported more BFRBs than the subclinical BFRB group $(M=2.11, S D=.85), t(94)=3.12, p=.02$. Further, results of Pearson's correlations indicated that the reported number of BFRBs was positively related to maladaptive hyperreactivity $(r=.20, p=$ $.02)$, experiential avoidance $(r=.23, p=.01)$, ability to maintain goal-directed behaviors when distressed $(r=$ $.17, p=.04)$, and ability to inhibit impulsive behavior when distressed $(p=.01)$. The number of reported BFRBs was unrelated to alexithymia $(p<.05)$.

\subsection{Alexithymia}

Results of a one-way MANOVA with BFRB group (i.e., BFRBD, subclinical BFRBs, and control) as the betweensubjects factor and the two alexithymia subscales of the DERS (i.e., DERS-Awareness and DERS-Awareness) as the dependent variables indicated that the BFRB groups did not differ on levels of alexithymia, $F(4,268)=$ 2.14, $p=.08$; Wilk's $\Lambda=.94 ; \eta_{\mathrm{p}}{ }^{2}=.03$. Means and standard errors on the DERS-Awareness and DERS-Clarity data are presented in Fig. 1.

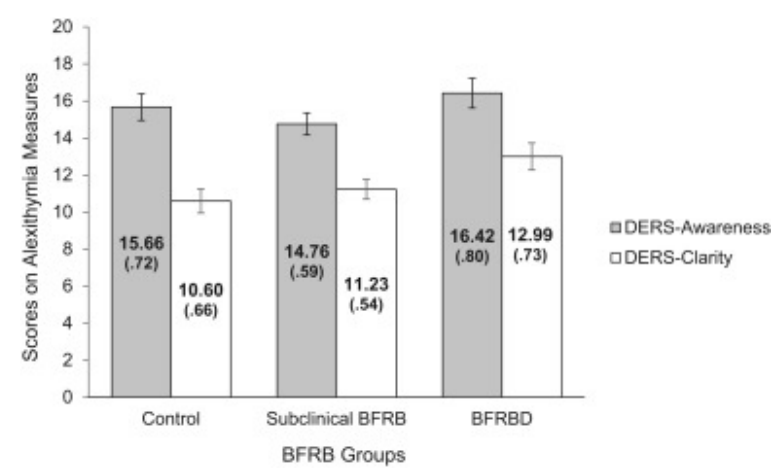

Fig. 1. Comparison of BFRB groups' mean alexithymia levels. Error bars represent standard error. DERS-Awareness = Difficulties in Emotion Regulation Scale: Awareness Subscale; DERS-Clarity = Difficulties in Emotion Regulation Scale: Clarity Subscale.

\subsection{Maladaptive emotional reactivity}

Results of a one-way ANOVA indicated that the BFRB groups reported significantly different levels of maladaptive emotional reactivity, $F(2,134)=9.47, p<.001, \eta_{p}{ }^{2}=.12$. Bonferroni post-hoc tests indicated that the BFRBD group reported higher levels of maladaptive hyperreactivity than the subclinical BFRB group, $p=.001$, and the control group, $p<.001$. The subclinical BFRB group and the control group did not report significantly different levels of hyperreactivity, $p=1.00$. Means and standard errors on the PAI-BOR-A data are presented and compared in Fig. 2. 


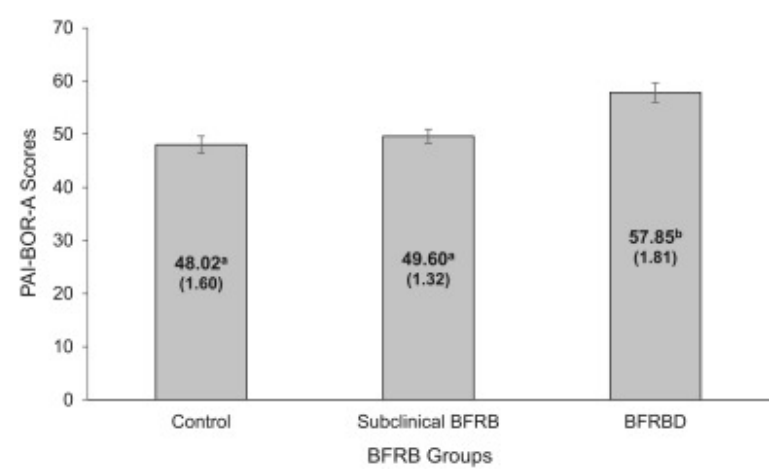

Fig. 2. Comparison of BFRB groups' mean maladaptive emotional reactivity levels. Error bars represent standard error. PAIBOR-A = Personality AssessmentInventory Borderline Features-Affective Instability subscale. Means sharing a superscript did not differ at $p<.05$ according to Bonferroni post-hoc analyses.

\subsection{Experiential avoidance}

Results of a one-way ANOVA indicated that the BFRB groups reported significantly different levels of experiential avoidance, $F(2,134)=17.50, p<.001, \eta_{p}^{2}=.21$. Bonferroni post-hoc tests indicated that the BFRBD group reported significantly higher levels of experiential avoidance than both the subclinical BFRB group, $p<.001$, and the control group, $p<.001$. The subclinical BFRB group and the control group did not report significantly different experiential avoidance levels, $p=1.00$. Means and standard errors on the AAQ-II data are presented and compared in Fig. 3.

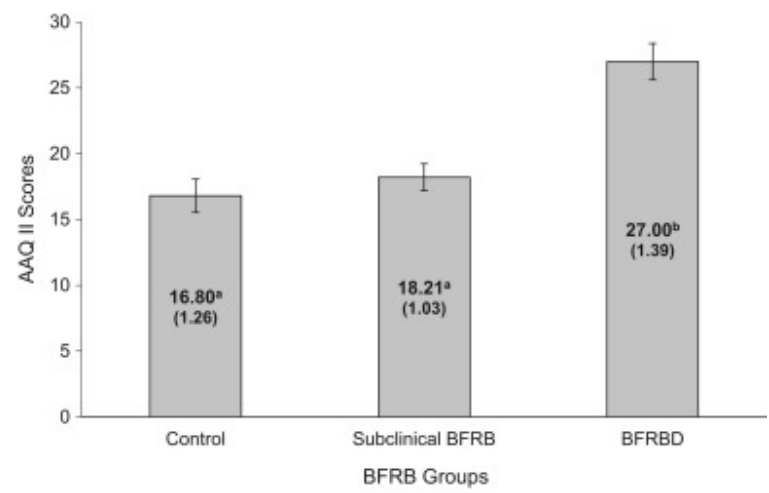

Fig. 3. Comparison of BFRB groups' mean experiential avoidance levels. Error bars represent standard error. AAQ-II = Acceptance and Action Questionnaire- Second Version. Means sharing a superscript did not differ at $p<.05$ according to Bonferroni post-hoc analyses.

\subsection{Ineffectual response inhibition when distressed}

Results of a one-way MANOVA with BFRB group (i.e., BFRBD, BFRB, and control) as the between-subjects factor and the two subscales of the DERS that measure response inhibition when distressed (i.e., DERS-Goals and DERS-Impulse) as the dependent variables indicated that the BFRB groups reported significant differences on ability to inhibit responses when distressed, $F(4,268)=6.12, p<.001 ;$ Wilk's $\Lambda=.84 ; \eta_{p}{ }^{2}=.08$. Follow-up univariate analyses indicated that the three groups significantly differed on the DERS-Goals subscale, $F(2,135)=$ $8.42, p<.001, \eta_{p}^{2}=.11$, and on the DERS-Impulse subscale, $F(2,135)=11.49, p<.001, \eta_{\mathrm{p}}^{2}=.15$.

Bonferroni post-hoc tests were used to further examine differences on the DERS-Goals and DERS-Impulse subscales. Post-hoc tests for the DERS-Goals subscale indicated that the BFRBD group reported having more difficulty maintaining goal-directed behavior when distressed than did both the subclinical BFRB group, $p=.002$, and the control group, $p=.001$. The subclinical BFRB group and the control group did not significantly differ 
from each other in terms of ability to maintain goal-directed behavior when distressed, $p=1.00$. Post hoc-tests for the DERS-Impulse subscale indicated that the BFRBD group reported having more difficulty maintaining control over their behaviors when distressed than did both the subclinical BFRB group, $p<.001$, and the control group, $p<.001$. The subclinical BFRB group and the control group did not significantly differ from each other in terms of ability to maintain control over behaviors when distressed, $p=1.00$. Means and standard errors on the DERS-Goals and DERS-Impulse data are presented and compared in Fig. 4.

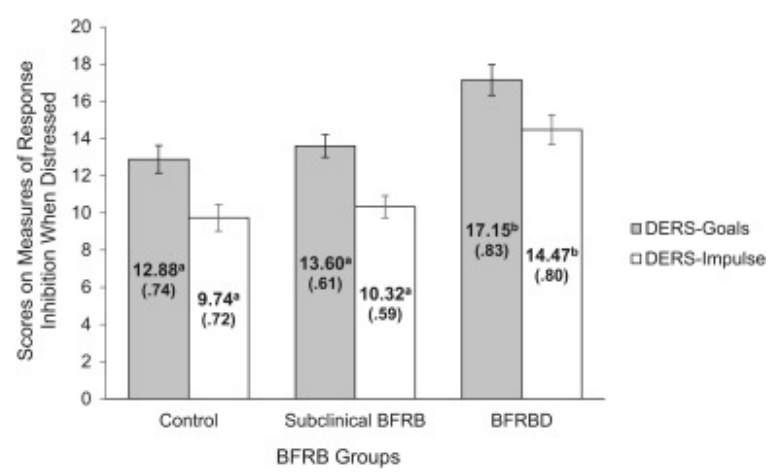

Fig. 4. Comparison of BFRB groups' mean experiential avoidance levels. Error bars represent standard error. DERS-Goals = Difficulties in Emotion Regulation Scale: Goals Subscale; DERS-Impulse = Difficulties in Emotion Regulation Scale: Impulse Subscale. Means sharing a superscript did not differ at $p<.05$ according to Bonferroni post-hoc analyses.

\subsection{Analyses controlling for comorbidity}

Three separate planned contrasts were conducted to examine whether the three groups (BFRBD, BFRB, Control) demonstrated the same differences on maladaptive emotional reactivity, experiential avoidance, and ineffectual response inhibition when distressed, when comorbidity was controlled for, as they did in the previously reported analyses. Results of these analyses did not change the pattern of results described above.

\subsection{Analyses controlling for number of reported BFRBs}

Three additional planned contrasts were conducted to examine whether the pattern of results pertaining to maladaptive emotional reactivity, experiential avoidance, and ineffectual response inhibition remained consistent when controlling for the number of reported BFRBs. Results of these analyses did not change the patterns described above.

\section{Discussion}

The current study was designed to extend understanding of ED in BFRBDs by (a) exploring the presence/nature of ED across multiple types of BFRBDs and (b) examining whether ED differentiates people with BFRBDs from those with subclinical BFRBs. Accordingly, the current study compared people with BFRBDs, people with subclinical BFRBs, and people unaffected by BFRBs on four ER deficits hypothesized to underlie ED (alexithymia, maladaptive emotional reactivity, experiential avoidance, and response inhibition when distressed). Consistent with hypotheses, participants with BFRBDs reported greater maladaptive hyperreactivity, experiential avoidance, and difficulty inhibiting responses when distressed in comparison to participants unaffected by BFRBs. Participants with BFRBDs also reported greater maladaptive hyperreactivity, experiential avoidance, and difficulty inhibiting responses when distressed in comparison to participants with subclinical BFRBs. Notably, these findings held, even when controlling for comorbidity status and total number of BFRBs, suggesting that differences were due to BFRBD status and not to artifacts of BFRBD status. These results mirror previous findings examining maladaptive hyperreactivity (Snorrason et al., 2010, Wetterneck et al., 2016), experiential avoidance (Arabatzoudis et al., 2017, Snorrason et al., 2010, Wetterneck et al., 2016), and response inhibition abilities when distressed (Arabatzoudis et al., 2017, Snorrason et al., 2010). Extending and clarifying previous findings, 
the current findings also suggest that, unlike people with BFRBDs, people with subclinical BFRBs do not demonstrate deficits in emotional reactivity, experiential avoidance, and ineffectual response inhibition when distressed, relative to unaffected controls.

Inconsistent with hypotheses, current results indicated that the BFRB groups did not report significantly different levels of alexithymia. These unexpected findings contrast previous findings from Roberts et al. (2016). Utilizing the same measure of alexithymia as the current study, Roberts et al. (2016) found that (a) people with BFRBs reported lower levels of emotional clarity than people without BFRBs and (b) people with and without BFRBs did not differ in terms of emotional awareness. The reasons for the discrepancy between the current findings and those of Roberts et al. (2016) are unclear. However, it may be worth noting that Roberts et al.'s (2016) BFRB sample potentially included people with subclinical BFRBs and people with BFRBDs. In addition, it may be worth noting that the average DERS-Awareness and DERS-Clarity subscale scores reported by both Roberts et al.'s (2016) BFRB and control groups were markedly lower than the average scores reported by the current study's BFRBD and control groups. In addition, the average DERS-Awareness and DERS-Clarity scores reported by Roberts et al.'s (2016) control group also appear to be markedly different from these subscales' norms (Gratz and Roemer, 2004).

Current results pertaining to alexithymia also conflict with findings from Çalikușu et al. (2002) and Snorrason et al. (2010). Sample differences may account for these discrepancies: in contrast to the current study's BFRBD sample composition, both Çalikuşu et al. (2002) and Snorrason et al.'s (2010)BFRBD samples solely consisted of clinical skin pickers. This difference may indicate that alexithymia deficits are not features of all BFRBD types; rather, alexithymia deficits may be most robust in clinical skin picking. We preliminarily tested this hypothesis by using $t$-tests to compare participants in the current BFRBD group who reported clinical skin picking with participants in the current subclinical BFRB group who reported subclinical skin picking on levels of emotional awareness and clarity. Similar to what was found by Calikuşu et al. (2002) and Snorrason et al. (2010), participants in the BFRBD group who reported clinical skin picking reported having marginally greater difficulty understanding emotions $(M=14.52, S E=1.23)$ than did participants in the subclinical BFRB group who reported subclinical skin picking $(M=11.57, S D=.84), p=.06$. However, these same participants did not differ on emotional awareness, $p=.35$.

Overall, results of the current study suggest that BFRBDs are characterized by ED. Furthermore, the current results suggest that ER deficits differentiate those whose BFRBs cause impairment (i.e., people with BFRBDs) from those whose BFRBs do not (i.e., people with subclinical BFRBs). Moreover, people with subclinical BFRBs do not appear to differ from people unaffected by BFRBs. These findings suggest that BFRBs may develop independent of ER deficits, but when a BFRB occurs in someone with deficits in emotional reactivity, experiential avoidance, and response inhibition when distressed, their risk of developing a BFRBD increases.

The current results may be useful for generating hypotheses about mechanisms by which BFRBs develop into BFRBDs. Previous research has shown that those with BFRBDs frequently perform BFRBs upon experiencing certain undesirable emotions (e.g., anxiety and boredom; Roberts et al., 2015; Teng et al., 2004). Building on these observations, the current results suggest that affected persons may be abnormally sensitive to experiencing certain emotions (i.e., maladaptive emotional reactivity) and, consequently, may experience these undesirable emotions frequently. As a result, affected persons may frequently implement BFRBs to regulate these undesirable emotions because of their unwillingness to experience these emotions (i.e., experiential avoidance) as well as their inability to maintain adaptive goal-directed behavior when experiencing these emotions (i.e., ineffectual response inhibition when distressed). Future research should investigate this conceptualization of BFRBDs. 
Furthermore, the current study may be useful for speculating about why research has shown that treatments such as Acceptance and Commitment Therapy (ACT; Hayes et al., 1999; Twohig et al., 2006), ACT-enhanced behavior therapy (AEBT; Twohig and Woods, 2004; Woods et al., 2006; Woods and Twohig, 2008), and Dialectical Behavior Therapy (DBT)-enhanced behavior therapy (Keuthen et al., 2012, Keuthen et al., 2011, Keuthen et al., 2010) effectively reduce BFRB severity and impairment. Specifically, the current results appear to support the hypothesis that these treatments reduce ER deficits which, in turn, leads to reductions in BFRB severity and impairment. Future research should investigate this possibility.

The current study had some limitations worth noting. First, the distribution of the BFRBD types (i.e., clinical hair pulling versus clinical skin picking) included in the current BFRBD sample was restricted. For instance, only 1 participant in the BFRBD group had clinical hair pulling, whereas 13 participants had clinical skin picking. Due to this restricted distribution, it is unclear whether the emotion regulation deficits identified in this study extend to all BFRBD types or simply to the BFRBD types that were most represented in the current BFRBD sample. The current sample's demographic uniformity (particularly in terms of age, ethnicity, and gender) is another limitation. A final limitation involves the fact that this study did not control for participants' negative affect (e.g., depression, anxiety, and neuroticism). Hypothetically, negative affect may be a factor that differentiates persons with BFRBDs from persons with subclinical BFRBs in the sense that one's level of negative affect may mediate whether they do or do not find their BFRB distressing/impairing. Because this study did not control for negative affect, the possibility that the BFRBD and subclinical BFRB groups differed on emotion regulation deficits because of systematic differences in negative affectivity levels cannot be dismissed. Future research exploring ER deficits in persons with BFRBDs should improve on these limitations.

Although the current study provides some evidence of the importance of ED in BFRBD status, several questions remain. First, the relationship between each of the outlined ER deficits and BFRB severity remains unclear. Second, the relationship between each of these ER deficits and BFRB substyles (e.g., automatic and focused) is fairly unknown despite conjecture that this relationship may be important (Arabatzoudis et al., 2017). Third, it is unclear why people with BFRBDs rigidly implement BFRBs as ER strategies and do not necessarily rigidly implement the ER strategies characteristic of other disorders (e.g., worry and rumination). Continued research on maladaptive ER's contribution to BFRBs and BFRBDs will be useful for answering such questions.

\section{Acknowledgements}

The authors would like to acknowledge the contribution of the Texas A\&M University Behavior Therapy and Research Laboratory undergraduate research assistants who assisted with creating databases for the current study.

\section{Role of the funding source}

This research did not receive any specific grant from funding agencies in the public, commercial, or not-for-profit sectors. Accordingly, aside from the authors, no other sources or persons contributed significantly to the study design, study protocol, statistical analyses, or final manuscript.

\section{References}

American Psychiatric Association, 2013 American Psychiatric Association The Diagnostic and Statistical Manual of Mental Disorders (5th ed.), American Psychiatric Association, Washington, DC (2013)

Arabatzoudis et al., 2017 T. Arabatzoudis, I.C. Rehm, M.Nedeljkovic, R. Moulding Emotion regulation in individuals with and without trichotillomania J. Obsessive Compuls. Relat. Disord., 12 (2017), pp. 8794, 10.1016/j.jocrd.2017.01.003 
Begotka et al., 2004 A.M. Begotka, D.W. Woods, C.T. Wetterneck The relationship between experiential avoidance and the severity of trichotillomania in a nonreferred sample J. Behav. Ther. Exp.

Psychiatry, 35 (2004), pp. 17-24, 10.1016/i.jbtep.2004.02.001

Berenbaum et al., 2003 H. Berenbaum, C. Raghavan, H.N. Le, L.L. Vernon, J.J. Gomez A taxonomy of emotional disturbances Clin. Psychol. Sci. Pract. (2003), 10.1093/clipsy/bpg011

Bond et al., 2011 F.W. Bond, S.C. Hayes, R.A. Baer, K.M.Carpenter, N. Guenole, H.K. Orcutt, T. Waltz, R.D. Zettle Preliminary psychometric properties of the Acceptance and Action Questionnaire-II: a revised measure of psychological inflexibility and experiential avoidance Behav. Ther., 42 (2011), pp. 676688, 10.1016/i.beth.2011.03.007

Çalikuşu et al., 2002 C. Çalikuşu, B. Yücel, A. Polat, C. Baykal Expression of anger and alexithymia in patients with psychogenic excoriation: a preliminary report (352-352) Int. J. Psychiatry Med., 32 (2002), 10.2190/22LB-HLOU-CPJP-WN6A

Diefenbach et al., 2002 G.J. Diefenbach, S. Mouton-Odum, M.A.Stanley Affective correlates of trichotillomania Behav. Res. Ther., 40 (2002), pp. 1305-1315, 10.1016/S0005-7967(02)00006-2

Diefenbach et al., 2008 G.J. Diefenbach, D.F. Tolin, S. Meunier, P. Worhunsky Emotion regulation and trichotillomania: a comparison of clinical and nonclinical hair pulling J. Behav. Ther. Exp.

Psychiatry, 39 (2008), pp. 32-41, 10.1016/j.jbtep.2006.09.002

Duke et al., 2009 D.C. Duke, D.K. Bodzin, P. Tavares, G.R.Geffken, E.A. Storch The phenomenology of hairpulling in a community sample J. Anxiety Disord., 23 (2009), pp. 1118-

1125, 10.1016/j.janxdis.2009.07.015

Flessner and Woods, 2006 C.A. Flessner, D.W. Woods Phenomenological characteristics, social problems, and the economic impact associated with chronic skin picking Behav. Modif., 30 (2006), pp. 944963, 10.1177/0145445506294083

Friedman and Miyake, 2004 N.P. Friedman, A. Miyake The relations among inhibition and interference control functions: a latent-variable analysis J. Exp. Psychol. Gen., 133 (2004), pp. 101-135, 10.1037/00963445.133.1.101

Gratz and Roemer, 2004 K.L. Gratz, L. Roemer Multidimensional assessment of emotion regulation and dysregulation: development, factor structure, and initial validation of the Difficulties in Emotion Regulation Scale J. Psychopathol. Behav. Assess., 26 (2004), pp. 41-

54, 10.1023/B:JOBA.000007455.08539.94

Gross, 2013 J.J. Gross Emotion regulation: conceptual and empirical foundations J.J. Gross (Ed.), Handbook of Emotion Regulation, Guilford Press, New York (2013), pp. 3-20

Hajcak et al., 2006 G. Hajcak, M.E. Franklin, R.F. Simons, N.J.Keuthen Hairpulling and skin picking in relation to affective distress and obsessive-compulsive symptoms J. Psychopathol. Behav. Assess., 28 (2006), pp. 177-185, 10.1007/s10862-005-9001-x

Hayes et al., 1999 S.C. Hayes, K.D. Strosahl, K.G. Wilson Acceptance and Commitment Therapy: an Experiential Approach to Behavior Change Guilford Press, New York (1999)

Hayes et al., 1996 S.C. Hayes, K.G. Wilson, E.V. Gifford, V.M.Follette, et al. Experiential avoidance and behavioral disorders: a functional dimensional approach to diagnosis and treatment J. Consult. Clin. Psychol., 64 (1996), pp. 1152-1168, 10.1037/0022-006X.64.6.1152

Hayes et al., 2009 S.L. Hayes, E.A. Storch, L. Berlanga Skin picking behaviors: an examination of the prevalence and severity in a community sample J. Anxiety Disord., 23 (2009), pp. 314-

319, 10.1016/i.janxdis.2009.01.008

Houghton et al., 2014 D.C. Houghton, S.N. Compton, M.P.Twohig, S.M. Saunders, M.E. Franklin, A.M. NealBarnett, L. Ely, M.R. Capriotti, D.W. Woods Measuring the role of psychological inflexibility in trichotillomania Psychiatry Res., 220 (2014), pp. 356-361, 10.1016/j.psychres.2014.08.003 
Keuthen et al., 2011

N.J. Keuthen, B.O. Rothbaum, M.J.Falkenstein, S. Meunier, K.R. Timpano, M.A. Jenike, S.S.Welch DBTenhanced habit reversal treatment for trichotillomania: 3- and 6-month follow-up results Depression Anxiety, 28 (2011), pp. 310-313, 10.1002/da.20778

Keuthen et al., 2012

N.J. Keuthen, B.O. Rothbaum, J. Fama, E.Altenburger, M.J. Falkenstein, S.E. Sprich, M. Kearns, S.Meunier, M.A. Jenike, S.S. Welch DBT-enhanced cognitive-behavioral treatment for trichotillomania: a randomized controlled trial J. Behav. Addict., 1 (2012), pp. 106-114, 10.1556/JBA.1.2012.003

Keuthen et al., 2010

N.J. Keuthen, B.O. Rothbaum, S.S. Welch, C. Taylor, M. Falkenstein, M. Heekin, C.A. Jordan, K.Timpano, S . Meunier, J. Fama, M.A. Jenike Pilot trial of dialectical behavior therapy-enhanced habit reversal for trichotillomania Depression Anxiety, 27 (2010), pp. 953-959, 10.1002/da.20732

Mansueto et al., 1997 C.S. Mansueto, R.M. Townsley Stemberger, A. McCombs Thomas, R. Goldfinger Golomb Trichotillomania: a comprehensive behavioral model Clin. Psychol. Rev. (1997), 10.1016/SO2727358(97)00028-7

Mennin et al., 2005 D.S. Mennin, R.G. Heimberg, C.L. Turk, D.M. Fresco Preliminary evidence for an emotion dysregulation model of generalized anxiety disorder Behav. Res. Ther., 43 (2005), pp. 1281-

1310, 10.1016/i.brat.2004.08.008

Morey, 2007 L.C. Morey Personality Assessment Inventory Professional Manual(2nd ed), Psychological Assessment Resources, Lutz (2007)

Morey, 1991 L.C. Morey Personality Assessment Inventory Professional Manual Psychological Assessment Resources, Odessa (1991)

Nock et al., 2008 M.K. Nock, M.M. Wedig, E.B. Holmberg, J.M.Hooley The Emotion Reactivity Scale: development, evaluation, and relation to self-injurious thoughts and behaviors Behav. Ther., 39 (2008), pp. 107-116, 10.1016/i.beth.2007.05.005

Nolen-Hoeksema et al., 2008 S. Nolen-Hoeksema, B.E. Wisco, S. Lyubomirsky Rethinking rumination Perspect. Psychol. Sci., 3 (2008), pp. 400-424, 10.1111/i.1745-6924.2008.00088.x

Norberg et al., 2007 M.M. Norberg, C.T. Wetterneck, D.W.Woods, C.A. Conelea Experiential avoidance as a mediator of relationships between cognitions and hair-pulling severity Behav. Modif., 31 (2007), pp. 367-381, 10.1177/0145445506297343

Oosterlaan et al., 1998 J. Oosterlaan, G.D. Logan, J.A. Sergeant Response inhibition in AD/HD, CD, comorbid $A D / H D+C D$, anxious, and control children: a meta-analysis of studies with the stop task J. Child Psychol. Psychiatry, 39 (1998), pp. 411-425, 10.1111/1469-7610.00336

Roberts et al., 2015 S. Roberts, K. O'Connor, F. Aardema, C.Bélanger The impact of emotions on body-focused repetitive behaviors: evidence from a non-treatment-seeking sample J. Behav. Ther. Exp.

Psychiatry, 46 (2015), pp. 189-197, 10.1016/j.jbtep.2014.10.007

Roberts et al., 2016 S. Roberts, K. O'Connor, F. Aardema, C.Bélanger, C. Courchesne The role of emotion regulation in body-focused repetitive behaviours Cogn. Behav. Ther., 9 (2016), p. e7, 10.1017/S1754470X16000039

Roberts et al., 2013 S. Roberts, K. O'Connor, C. Bélanger Emotion regulation and other psychological models for body-focused repetitive behaviors Clin. Psychol. Rev., 33 (2013), pp. 745-

762, 10.1016/j.cpr.2013.05.004

Rothbaum and Ninan, 1994 B.O. Rothbaum, P.T. Ninan The assessment of trichotillomania Behav. Res. Ther., 32 (1994), pp. 651-662, 10.1016/0005-7967(94)90022-1

Rufer et al., 2014 M. Rufer, T. Bamert, R. Klaghofer, S. Moritz, L. Schilling, S. Weidt Trichotillomania and emotion regulation: is symptom severity related to alexithymia? Psychiatry Res., 218 (2014), pp. 161165, 10.1016/j.psychres.2014.03.029 
Sheehan et al., 1998

D.V. Sheehan, Y. Lecrubier, K.H. Sheehan, P. Amorim, J. Janavs, E. Weiller, T. Hergueta, R. Baker, G.C. Du nbar The Mini-International Neuropsychiatric Interview (M.I.N.I.): The development and validation of a structured diagnostic psychiatric interview for DSM-IV and ICD-10 J. Clin. Psychiatry (1998), pp. 2223, 10.1016/S0924-9338(99)80239-9

Snorrason et al., 2012 Í. Snorrason, E.J. Ricketts, C.A. Flessner, M.E. Franklin, D.J. Stein, D.W. Woods Skin picking disorder is associated with other body-focused repetitive behaviors: findings from an internet study Ann. Clin. Psychiatry, 24 (2012), pp. 292-299

Snorrason et al., 2010 í. Snorrason, J. Smari, R.P. Olafsson Emotion regulation in pathological skin picking:

findings from a non-treatment seeking sample J. Behav. Ther. Exp. Psychiatry, 41 (2010), pp. 238245, 10.1016/i.jbtep.2010.01.009

Stanley et al., 1995 M.A. Stanley, J.W. Borden, S.G. Mouton, J.K.Breckenridge Nonclinical hair-pulling: affective correlates and comparison with clinical samples Behav. Res. Ther., 33 (1995), pp. 179-

186, 10.1016/0005-7967(94)E0018-E

Stein et al., 2008 D.J. Stein, C.A. Flessner, M. Franklin, N.J.Keuthen, C. Lochner, D.W. Woods Is trichotillomania a stereotypic movement disorder? An analysis of body-focused repetitive behaviors in people with hair-pulling Ann. Clin. Psychiatry, 20 (2008), pp. 194-198, 10.1080/10401230802435625

Stein et al., 2007 M.B. Stein, J.H. Pinsker-Aspen, M.J.Hilsenroth Borderline pathology and the Personality Assessment Inventory (PAI): an evaluation of criterion and concurrent validity J. Personal. Assess., 88 (2007), pp. 81-89, 10.1207/s15327752jpa8801 11

Taylor, 2000 G.J. Taylor Recent developments in alexithymia theory and research Can. J. Psychiatry (2000), 10.1177/070674370004500203

Teng et al., 2004 E.J. Teng, D.W. Woods, B.A. Marcks, M.P.Twohig Body-focused repetitive behaviors: the proximal and distal effects of affective variables on behavioral expression J. Psychopathol. Behav. Assess., 26 (2004), pp. 55-64, 10.1023/B:JOBA.0000007456.24198.e4

Teng et al., 2002 E.J. Teng, D.W. Woods, M.P. Twohig, B.A.Marcks Body-focused repetitive behavior problems prevalence in a nonreferred population and differences in perceived somatic activity Behav. Modif., 26 (2002), pp. 340-360, 10.1177/0145445502026003003

Twohig et al., 2006 M.P. Twohig, S.C. Hayes, A. Masuda A preliminary investigation of acceptance and commitment therapy as a treatment for chronic skin picking Behav. Res. Ther., 44 (2006), pp. 15131522, 10.1016/j.brat.2005.10.002

Twohig and Woods, 2004 M.P. Twohig, D.W. Woods A preliminary investigation of acceptance and commitment therapy and habit reversal as a treatment for trichotillomania Behav. Ther., 35 (2004), pp. 803-820, 10.1016/S0005-7894(04)80021-2

Wetterneck et al., 2016 C.T. Wetterneck, E.B. Lee, C.A. Flessner, R.C. Leonard, D.W. Woods Personality characteristics and experiential avoidance in Trichotillomania: results from an age and gender matched sample J. Obsessive Compuls. Relat. Disord., 8 (2016), pp. 64-69, 10.1016/i.jocrd.2015.12.003

Woods and Twohig, 2008 D.W. Woods, M.P. Twohig Trichotillomania: An ACT-enhanced Behavior Therapy Approach Therapist Guide Oxford University Press, New York (2008)

Woods et al., 2006 D.W. Woods, C.T. Wetterneck, C.A. Flessner A controlled evaluation of acceptance and commitment therapy plus habit reversal for trichotillomania Behav. Res. Ther., 44 (2006), pp. 639$656, \underline{10.1016 / j . b r a t .2005 .05 .006}$

(C) 2017 Elsevier B.V. All rights reserved. 